Canadian

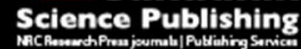

Canadian Journal of Microbiology Revue canadienne de de microbiologie

\title{
Antigenic and Genetic Characterization of Bordetella pertussis recovered from Quebec, Canada, 2002-2014: detection of a genetic shift
}

\begin{tabular}{|r|l|}
\hline Journal: & Canadian Journal of Microbiology \\
\hline Manuscript ID & cjm-2015-0781.R1 \\
\hline Manuscript Type: & Article \\
\hline Date Submitted by the Author: & 05-Jan-2016 \\
\hline Kist of Authors: & $\begin{array}{l}\text { Shuel, Michelle; Public Health Agency of Canada, National Microbiology } \\
\text { Laboratory } \\
\text { Lefebvre, Brigitte; Laboratoire de santé publique du Québec, } \\
\text { Whyte, Kathleen; Public Health Agency of Canada, National Microbiology } \\
\text { Laboratory } \\
\text { Hayden, Kristy; Public Health Agency of Canada, National Microbiology } \\
\text { Laboratory } \\
\text { De Serres, Gaston; Institut National de Sante Publique du Quebec } \\
\text { Brousseau, Nicholas; Institut National de Sante Publique du Quebec } \\
\text { Tsang, Raymond S.W.; CNS Infection Division and Vaccine Preventable } \\
\text { Bacterial Diseases Division, }\end{array}$ \\
\hline Keyword: & \begin{tabular}{l} 
Bordetella pertussis, pertactin, genotyping \\
\hline
\end{tabular} \\
\hline
\end{tabular}


1 Antigenic and Genetic Characterization of Bordetella pertussis recovered from Quebec, Canada,

2 2002-2014: detection of a genetic shift

3

4 Michelle Shuel $^{1}$, Brigitte Lefebvre ${ }^{2}$, Kathleen Whyte ${ }^{1}$, Kristy Hayden ${ }^{1}$, Gaston De Serres ${ }^{3,4}$,

5 Nicholas Brousseau ${ }^{3}$, and Raymond S.W. Tsang ${ }^{1}$,

6

7 Public Health Agency of Canada, National Microbiology Laboratory, Winnipeg, Manitoba,

8 Canada $^{1}$; Laboratoire de santé publique du Québec, Sainte-Anne-de-Bellevue, Québec, Canada ${ }^{2}$;

9 Institut National de Sante Publique du Québec, Québec, Canada ${ }^{3}$; and Laval University, Québec, 10 Canada ${ }^{4}$.

11

12

13 Corresponding Author:

14 Dr. Raymond Tsang

15 Public Health Agency of Canada

16 National Microbiology Laboratory

17 Laboratory for Syphilis Diagnostics and Vaccine Preventable Bacterial Diseases

181015 Arlington Street

19 Winnipeg, Manitoba, Canada R3E 3R2

20 Phone: 204-789-6020 Fax: 204-789-2018

21 Email: raymond.tsang@phac-aspc.gc.ca 
2 Abstract

3 Despite vaccination, cyclical peaks of Bordetella pertussis incidence rates are still observed in

4 Canada and other developed countries, making pertussis one of the most prevalent vaccine

5 preventable bacterial diseases. In the post-acellular vaccine era, evolution of bacterial strains has

6 resulted in strains with altered vaccine antigens. Previous Canadian studies have focused on

7 isolates mainly from the provinces of Ontario and Alberta, with only small numbers of isolates

8 from other provinces. Therefore, in this study, we examined a larger sample $(n=52)$ of isolates

9 from Québec, Canada between 2002 and 2014. Isolates were characterized by serotype, sequence

10 type and prevalence of pertactin-deficiency. The Québec isolates shared similar characteristics

11 with other Canadian isolates as well as isolates circulating globally. Although pertactin-deficient

12 isolates were not present, a significant shift in sequence type was observed in more recent years.

13 This study highlights the importance of continually monitoring disease causing isolates in order

14 to track evolutionary trends and gain a better understanding of the molecular epidemiology of

15 pertussis in Canada.

16

17 Keywords: Bordetella pertussis, pertactin, genotyping 
2 Whooping cough, caused by the bacterium Bordetella pertussis, became a nationally notifiable 3 disease in Canada in 1924 (Varughese 1985). Prior to the introduction of the vaccine, cyclical

4 increase in incidence was occurring every 2 to 5 years and the average incidence rate was 156 5 cases per 100,000 in the five years before vaccination (Smith et al. 2014). Vaccination began in 61943 using a fluid whole cell vaccine, which was replaced in the 1980s by the adsorbed whole

7 cell vaccine manufactured by Connaught. The introduction of this poorly protective vaccine was

8 rapidly followed by a large resurgence of pertussis. This product was replaced in 1997-98 by the

9 less reactogenic and more efficacious acellular vaccines. As a result of vaccination, pertussis

10 incidence decreased dramatically, reaching a record low in Canada of 2 cases per 100,000 in

112011 (Smith et al. 2014). Despite better vaccine effectiveness and extensive coverage, periodic

12 outbreaks still occur and the last peak in Québec was observed in 2012 (Smith et al. 2014).

13 During that year, the national rate of pertussis jumped seven fold (13.9 cases per 100,000) and 9 14 out of 13 Canadian provinces and territories reported increased disease activity with a large 15 outbreak in the province of New Brunswick which accounted for $1 / 3$ of the cases nationally 16 (Smith et al. 2014).

Acellular vaccines have now replaced whole cell vaccines in many industrialized countries. They are made up of purified pertussis proteins and contain between 1 and 5 of the following

20 components: pertussis toxin (Ptx), pertactin (Prn), filamentous haemagglutinin (Fha) and

21 fimbriae types 2 and 3 (Fim 2 and Fim 3). In Québec, the 5 (Ptx+Fha+Prn+Fim 2/3) components

22 acellular pertussis vaccine has mostly been used in the past decade but the 3 (Ptx+Fha+Prn)

23 component vaccine is also offered. The schedule consists of 3 primary doses given at 2, 4, and 6 
1 months, followed by booster shots given at 18 months, another between 4 and 6 years of age, and

2 again between 14 and 16 years of age. Since 2013, a single booster is recommended after 18

3 years of age (MSSS 2013). With vaccination coverage rates $>95 \%$ with $\geq 3$ doses at 24 months

4 of age (Boulianne et al. 2014), pertussis incidence rates have been relatively low in Québec in

5 the past 15 years. This is different from the pertussis resurgence (larger number of cases than

6 expected, given the periodic variability of pertussis disease, when compared to previous cycles in

7 the same setting) observed in several countries, including Australia, Portugal, USA and UK

8 (WHO 2015). For example, California noticed in 2010 the highest number of pertussis cases in

9 more than 60 years, including $>9000$ cases, 809 hospitalizations and 10 deaths (Winter 2012). In

10 2012, a large UK outbreak saw nearly 10,000 cases, including 14 deaths in infants under the age

11 of 3 months (Sealey et al. 2015). This resurgence of pertussis has been linked to several factors:

12 1) waning immunity, especially in the adolescent/adult populations; 2) improved laboratory

13 methods, including the use of PCR for diagnosis; 3) lower efficacy of the acellular vaccines

14 compared to whole cell vaccines; 4) higher rates of non-vaccination in recent years and 5)

15 evolution of the B. pertussis bacterium (Guiso 2014, Jakinovich and Sood 2014, Mooi et al.

16 2014, Belcher and Preston 2015).

17

18 Evolution of the $B$. pertussis bacterium over the years has resulted in a divergence between

19 currently circulating strains versus the strains used in vaccine production (Bart et al. 2014). It has

20 also led to the emergence of strains with mutations in cell surface protein encoding genes,

21 resulting in strains with altered vaccine antigens (van Gent et al. 2015). Reports of strains with

22 increased fitness due to the $p t x P 3$ type mutations in the $p t x$ promoter region emerged in the

23 1980s and now predominate worldwide (Mooi et al. 2009, Advani et al. 2011, Octavia et al. 
1 2012, Schmidtke et al. 2012, Shuel et al. 2013, Pawloski et al. 2014). These isolates are thought

2 to have increased levels of pertussis toxin, however this has not been confirmed at the

3 transcriptomic level (King et al. 2013, de Gouw et al. 2014, Bouchez et al. 2015). Isolates

4 lacking cell surface proteins Fha, Ptx, Fim and Prn have also been identified (Bouchez et al.

5 2009, Hegerle et al. 2012, Shuel et al. 2013, Pawloski et al. 2014, Zeddeman et al. 2014). Isolates

6 lacking Ptx, Fha or Fim are relatively uncommon compared to those lacking Prn (Zeddeman et

7 al. 2014). The Prn-deficient strains are now a cause for global concern as they have emerged in

8 areas such as the US, Japan, Australia and several European countries (Pawloski et al. 2014,

9 Otsuka et al. 2012, Lam et al. 2014, Zeddeman et al. 2014). In addition to its prevalence, Prn-

10 deficient strains appear to have a selective advantage over Prn-positive strains, especially in the

11 context of acellular pertussis vaccination. Prn-deficient strains were found more often from

12 patients who have received acellular pertussis vaccine when compared to unvaccinated patients

13 (Martin et al. 2015). Also Prn-deficient strains caused longer colonisation than Prn-positive

14 strains in acellular pertussis vaccine immunised mice (Hegerle et al. 2012).

17 A previous study, focussing on Canada's most populous province of Ontario, identified 1

18 predominant clone (ST-1) that was seen in 83.5\% of isolates (Shuel et al. 2013). Another study

19 (Tsang et al. 2014) which included isolates from 8 out of 13 Canadian provinces and territories,

20 identified Prn-deficient isolates in only 2 provinces:Ontario (7/138 isolates) and Alberta (5/56

21 isolates). However, in that study, only 3 isolates recovered in 2008 from Québec were included.

22 Therefore, in this study we examined a larger sample $(n=52)$ over more than 10 years in order

23 to ascertain the prevalence or absence of Prn-deficient isolates in Québec. Here we present the 
1 characterization of $B$. pertussis isolates collected between 2002 and 2014 from the province of

2 Québec: Canada's largest province in land area and second largest province based on population.

4 There were 52 B. pertussis isolates sent to the National Microbiology Laboratory (NML) for the 5 purpose of this study. Isolates were collected by the provincial reference laboratory of Québec,

6 Laboratoire de sante publique du Québec (LSPQ), between 2002 and 2014 and represented all

7 years, with the exception of 2011 and 2013. There were 5 isolates for the years 2002, 2003,

8 2004, 2005, 2006 and 2009; 2 isolates for 2007 and 2010; 7 isolates for 2008, 10 isolates for

92012 and 1 isolate for 2014. Isolates were serotyped by indirect whole-cell ELISA (Tsang et al,

10 2005) and genotyped by partial sequencing of the following virulence factor genes: pertussis

11 toxin subunit 1 (ptxS1), pertactin (prn), fimbriae 3 (fim3), filamentous haemagglutinin (fhaB) and

12 the pertussis toxin promoter region $(p t x P)$ in order to determine sequence type (ST) (Shuel et al,

13 2013). In order to identify Prn-deficient isolates, complete sequencing of the prn gene was

14 performed on all 52 isolates and Western blot for the detection of pertactin was carried out on 51

15 isolates according to procedures previously described (Tsang et al, 2014).

16

17 All 52 isolates were found to belong to the Fim 3 serotype. Genotyping found that the majority 18 of isolates belonged to ST-1 ( $\mathrm{n}=34,65.4 \%)$ characterized by $p t x S 1 A$, prn 2, fim $3 B$, fhaB 1 and ptxP3, followed by ST-2 (n=12, 23\%) (ptxS1A, prn2, fim3A, fhaB 1, ptxP3), ST-8 (n=4, 7.7\%)

20 (ptxS1A, prn9, fim3B, fhaB 1, ptxP3), and the 2 remaining isolates belonged to ST-4 (ptxS1A, 21 prn1, fim 3A, fhaB1,ptxP1) and ST-7 (ptxS1A, prn3, fim3B, fhaB1, ptxP3). There were no Prn-

22 deficient isolates identified. All isolates were found to contain an intact prn gene based on 
1 complete prn gene sequence analysis and all 51 isolates tested by Western immunoblot were

2 found to produce the Prn protein.

4 Sequence types 1 and 2 differ only in their fim 3 allele type and were the most common STs

5 identified in this study as well as in previous Canadian studies from the provinces of Ontario and

6 Alberta (Shuel et al. 2013, Simmonds et al. 2014). Ontario had the highest frequency of ST-1

7 isolates at $83.5 \%$, compared to $77 \%$ and $65.4 \%$ from Alberta and Québec, respectively. ST-2 was

8 found in frequencies of 10.9\%, 19.2\% and 23\% in Ontario, Alberta and Québec, respectively. In

9 the Québec isolates, there was a significant shift from ST-1 (fim $3 B$ ) to ST-2 (fim 3A) in more recent years $(p<0.01$; Table 1). The percentage of ST-2 isolates increased from $4 \%(1 / 25)$ in

11 isolates collected prior to 2006 to $40.7 \%$ (11/27) after 2006 while for the same period, ST-1

12 isolates decreased from $92.0 \%(23 / 25)$ to $40.7 \%(11 / 27)$.

14 Circulating B. pertussis clones from Australia, the US and Europe also share similar

15 characteristics with Canadian isolates (Octavia et al. 2012, Pawloski et al. 2014, van Gent et al.

16 2015). A study on 466 B. pertussis isolates from 13 European countries found that the Fim 3

17 serotype and prn2 and ptxP3 allele types were dominant in all countries except Poland, where the

18 whole cell vaccine is still being used (van Gent et al. 2015). In contrast, Polish isolates were mainly typed as serotype Fim 2, ptxPl and either prn 1 or prn2. A slight shift from ST-1 (fim3B

20 or fim3-2) to ST-2 (fim3A or fim3-1) was also apparent, although not quite as pronounced as

21 what was noted in Québec. Allele type fim3B was seen in 59\% of European isolates between

222002 and 2006 and fim $3 A$ was observed in 41\%. Between 2007 and 2012, 42\% were fim $3 B$ and

$2356 \%$ fim $3 A$ (van Gent et al. 2015). 
2 Strains lacking Prn expression arise from a multitude of different mutational events, including

3 disruption of the prn gene with insertion sequences IS481 or IS 1002;

4 mutations/inversions/deletions in the promoter region or within the gene itself; single nucleotide

5 polymorphisms leading to premature stop codons and complete deletion of the entire prn gene

6 (Zeddeman et al. 2014, Lam et al. 2014, Pawloski et al. 2014, Tsang et al. 2014). First identified

7 in the US in 1994, Prn-deficient strains have become increasingly more common, especially in

8 Australia and the US (Lam et al. 2014, Pawloski et al. 2014). Prn-deficient B. pertussis increased

9 rapidly in Australia, going from 5\% in 2008 to $78 \%$ in 2012 (Lam et al. 2014) and in the US,

10 Prn-deficient strains were responsible for $>50 \%$ of the cases in 2012 (Pawloski et al. 2014). Prn-

11 deficient strains are not quite as prevalent in Europe, ranging from $0 \%$ up to $25 \%$, depending on

12 country, with an overall average of 3.4\% based on 414 isolates tested between 2007 and 2012

13 (Zeddeman et al. 2014). A previous Canadian report on 224 isolates from 1994 to 2013 found

14 that 5.4\% were Prn-deficient and all were isolated in either 2011 or 2012 from the non-

15 neighboring provinces of Alberta and Ontario (Tsang et al. 2014). However, only very small

16 numbers of isolates from the provinces of British Columbia $(n=12)$, Manitoba $(n=3)$,

17 Saskatchewan $(n=8)$, Québec $(n=3)$, and Nova Scotia $(n=3)$ were included in that study; and

18 therefore, would not be definitive in terms of prevalence of such isolates in these provinces. The 19 present study includes 10 isolates from 2012 and no isolates from 2011.

20

21 It has been suggested that the lack of Prn production is a direct result of selection pressure

22 mediated by the acellular vaccines; and genes encoding vaccine antigens have been shown to

23 evolve at a significantly faster rate compared to genes encoding other cell surface proteins 
1 (Sealey et al. 2015). This supports the idea that selection pressure may be the driving force

2 behind evolution of $B$. pertussis. Although Prn-deficient isolates present a better fitness in

3 subjects primed or vaccinated with acelular pertussis vacines (Hegerle et al. 2012, Martin et al.

4 2015), acellular vaccines do provide good protection in the short term especially when the

5 coverage is high. In Australia and the US, where a resurgence of pertussis was observed (WHO

6 SAGE pertussis Working Group 2014), an increasing proportion of strains are Prn-deficient.

7 Interestingly, there is no strong evidence of pertussis resurgence in Canada (WHO SAGE

8 Pertussis Working Group 2014, Smith et al. 2014) where Prn-deficient stains are uncommon.

9 Although this does not represent any evidence of a causal association and some countries, such

10 as the UK, observed pertussis resurgence without any increase in Prn-deficient strains (Sealy

11 2015). Thus further work regarding the impact of Prn deficiency on vaccine effectiveness is

12 required. Another possible hypothesis for the absence of pertussis resurgence in Québec and

13 most other Canadian provinces is the use of the poorly protective adsorbed Connaught whole cell

14 pertussis vaccine (De Serres 1996, Bettinger 2007) and the extensive transmission of disease in

15 the 1990s (Ntezayabo 2003), leading to some degree of protective immunity at the population

16 level.

17

18 Surveillance of $B$. pertussis in Québec and Canada is hindered by the fact that there is no formal

19 program in place and isolates are not routinely sent to the LSPQ nor to the NML for

20 characterization. Consequently, one limit of this study is the number of isolates which

21 represented only about 5\% of culture positive samples in Québec from 2002-2014. Also, more

22 and more laboratories are relying on PCR for diagnosis and not culturing for the bacterium, 
1 rendering it increasingly difficult to document the evolving strain characteristics in pertussis

2 cases in Canada.

3

4 Based on reports so far, many of the Canadian isolates appear to share similar characteristics

5 with each other as well as with $B$. pertussis isolates circulating worldwide. However, this is the

6 first report of a significant shift in ST over time. While Prn-deficient isolates were interestingly

7 absent from the Québec isolates studied, the number of isolates examined was still small and the

8 potential for rapid increase still exists, such as what has been seen in Australia, the US and other

9 Canadian provinces (Pawloski et al. 2014, Lam et al. 2014, author's unpublished data).

10 Therefore, it is important to continually monitor future isolates for both of these trends and

11 maintain culture capability in Québec and Canada in order for strain characterization to be done

12 either periodically or when required. It is only when strain characterization is coupled with

13 epidemiological investigation and clinical information that a more comprehensive view of

14 pertussis can be achieved in Québec and in Canada.

15

16 
2 Acknowledgements

3

4 The authors would also like to thank the staff at the National Microbiology Laboratory's

5 Genomics Core Facility for the primer synthesis and DNA sequencing work, Saul Deng for some

6 of the Western blot work, as well as students who contributed to this project: Mitchele Demelo,

7 Jessica Wong, William Hoang and Sarah Moore. 


\section{References}

4 Advani, A., Gustafsson, L. Ahren, C., Mooi, F.R., and Hallander, H.O. 2011. Appearance of fim3

5 and ptxP3-Bordetella pertussis strains, in two regions of Sweden with different vaccination

6 programs. Vaccine. 29(18):3448-425.

Bart, M.J., Harris, S.R., Advani, A., Arakawa, Y., Bottero, D., Bouchez, V., Cassiday, P.K.,

9 Chiang, C.S., Dalby, T., Fry, N.K., Gaillard, M.E., van Gent, M., Guiso, N., HJallander, H.O.,

Harvill, E.T., He, Q., van der Heide, H.G., Heuvelman, K., Hozbor, D.R., Kamachi, K.,

11 Karataev, G.I., Lan, R., Lutynska, A., Maharjan, R.P., Mertsola, J., Miyamura, T., Octavia, S.,

12 Preston, A., Quail, M.A., Sintchenko, V., Stefanelli, P., Tondella, M.L., Tsang, R.S., Xu, Y.,

13 Yao, S.M., Zhang, S., Parkhill, J. and Mooi, F.R. 2014. Global population structure and evolution of Bordetella pertussis and their relationship with vaccination. MBio. 5(2):e01074.

Belcher, T. and Preston, A. 2015. Bordetella pertussis evolution in the (functional) genomics era.

17 Pathog. Dis. 73(8):ftv064 changing from whole-cell to acellular pertussis vaccine on the epidemiology of hospitalized children with pertussis in Canada. The Pediatric Infectious Disease Journal. 26(1):31-35. 
1 Bouchez, V., Brun, D., Cantinelli, T., Dore, G., Njamkepo, E. and Guiso, N. 2009. First report

2 and detailed characterization of $B$. pertussis isolates not expressing pertussis toxin or pertactin.

3 Vaccine. 27(43):675-82.

4

5 Bouchez, V., Hegerle, N., Strati, F., Njamkepo, E. and Guiso, N. 2015. New data on vaccine

$6 \quad$ antigen deficient Bordetella pertussis isolates. Vaccines. 3(3) :751-70.

7

8 Boulianne, M., Audet, D., Ouakki, M. 2014. Enquête sur la couverture vaccinale des enfants de 1 9 et 2 ans au Québec en 2014. Québec: Institut national de sante publique du Quebec, 151 p.

11 De Gouw, D., Hermans, P.W., Bootsma, H.J., Zomer, A., Heuvelman, K., Diavatopolous, D.A. 12 and Mooi, F.R. 2014. Differentially expressed genes in Bordetella pertussis strains belonging to 13 a lineage which recently spread globally. PLoS One. 9(1):e84523.

14

15 De Serres, G., Boulianne, N., Duval, B., Déry, P., Rodriguez, A.M., Massé, R, Halperin S. 1996. 16 Effectiveness of a whole cell pertussis vaccine in child-care centers and schools. Ped Infect Dis J $17 \quad 15: 519-524$.

18

19 Guiso, N. 2014. Pertussis vaccination and whooping cough: and now what? Expert Rev 20 Vaccines. 13(10):1163-5.

21 
1 Hegerle, N., Paris, A.-S., Brun, D., Dore, G., Njamkepo, E., Guillot, S., and Guiso, N. 2012.

2 Evolution of French Bordetella pertussis and Bordetella parapertussis isolates: increase of

3 Bordetellae not expressing pertactin. Clin. Microbiol. Infect. 18(9):e340-6.

4

5 Jakinovich, A. and Sood, S.K. 2014. Pertussis: still a cause of death, seven decades into

6 vaccination. Curr. Opin. Pediatr. 26(5):597-604.

7

8 Kallonen, T. and He, Q. 2009. Bordetella pertussis strain variation and evolution

9 postvaccination. Expert. Rev. Vaccines. 8(7):863-75.

10

11 King, A.J., van der Lee, S., Mohangoo, A., van Gent, M., van der Ark, A. and van de

12 Waterbeemd, B. 2013. Genome-wide gene expresstion analysis of Bordetella pertussis isolates

13 associated with a resurgence in pertussis: elucidation of factors involved in the increased fitness

14 of epidemic strains. PLoS One. 8(6):e66150.

15

16 Lam, C., Octavia, S., Ricafort, L., Sintchenko, V., Gilbert, G.L., Wood, N., McIntypre, P.,

17 Marshall, H., Guiso, N., Keil, A.D., Lawrence, A., Robson, J., Hogg, G. and Lan, R. 2014. Rapid

18 increase in pertactin-deficient Bordetella pertussis isolates, Australia. Emerg. Infect. Dis.

$1920(4): 626-33$.

20

21 Martin, S.W., Pawloski, L., Williams, M., Weening, K., DeBolt, C., Qin, X., et al. 2015.

22 Pertactin-negative Bordetella pertussis strains: evidence for a possible selective advantage. Clin.

23 Infect. Dis. 60 : 223-227. 
2 Ministère de la Santé et des Services sociaux du Québec (MSSS). 2013. Protocole

3 d'immunisation du Québec. Quebec : Direction des communications, ministère de la Santé et des

4 Services sociaux; 2013, 505 p.

5

6 Mooi, F.R., van Loo, I.H., van Gent, M., He, Q., Bart, M.J., Heuvelman, K.J., de Greef, S.C.,

7 Diavatopoulos, D., Teunis, P., Nagelkerke, M., and Mertsola, J. 2009. Bordetella pertussis

8 strains with increased toxin production associated with pertussis resurgence. Emerg. Infect. Dis.

$9 \quad$ 15(8): 1206-13.

10

11 Mooi, F.R., Van Der Maas, N.A., and De Melker, H.E. 2014. Pertussis resurgence: waning

12 immunity and pathogen adaptation - two sides of the same coin. Epidemiol Infect. 142(4):685-

1394.

14

15 Ntezayabo, B., De Serres, G., and Duval, B. 2003. Pertussis resurgence in Canada largely due to 16 a cohort effect. Ped Infect Dis J 22(1):22-27.

17

18 Octavia, S., Sintchenko, V., Gilbert, G.L., Laswrence, A., Keil, A.D., Hogg, G. and Lan, R.

19 2012. Newly emerging clones of Bordetella pertussis carrying prn2 and ptxP3 alleles implicated

20 in Australian pertussis epidemic in 2008-2010. J. Infect. Dis. 205(8):1220-4.

21 
1 Otsuka, N., Han, H.J., Toyoizumi-Ajisaka, H., Nakamura, Y., Arakawa, Y., Shibayama, K., and

2 Kamachi, K. 2012. Prevalence and genetic characterization of pertactin-deficient Bordetella

3 pertussis in Japan. PLoS One. 7(2):e31985.

4

5 Pawloski, L.C., Queenan, A.M., Cassiday, P.K., Lynch, A.S., Harrison, M.J., Shang, W.,

6 Williams, M.M., Bowden K.E., Burgos-Rivera, B., Qin, X., Messonnier, N., and Tondella, M.L.

7 2014. Prevalence and molecular characterization of pertactin-deficient Bordetella pertussis in the

8 United States. Clin. Vaccine. Immunol. 21(2):119-25.

9

10 Schmidtke, A.J., Boney K.O., Martin, S.W., Skoff, T.H., Tondella, M.L,and Tatti, K.M. 2012.

11 Population diversity among Bordetella pertussis isolates, United States, 1935-2009. Emerg.

12 Infect. Dis. 18(8):1248-55.

14 Sealey, K.L., Harris, S.R., Fry, N.K., Laurence, D.H., Gorringe, A.R., Parkhill, J. and Preston, A.

15 2015. Genome analysis of isolates from the United Kingdon 2012 pertussis outbreak reveals that

16 vaccine antigen genes are unusually fast evolving. J. Infect. Dis. 212(2):294-301.

17

18 Shuel, M., Jamieson, F.B., Tang, P., Brown, S., Farrell, D., Martin, I., Stoltz, J. and Tsang, R.S.

19 2013. Genetic analysis of Bordetella pertussis in Ontario, Canada reveals one predominant

20 clone. Int. J. Infect. Dis. 17(6):e413-7.

21 
1 Simmonds, K., Fathima, S., Chui, L., Lovgren, M., Shook, P., Tyrell, G., Tsang, R. and Drews,

2 S.J. 2014. Dominance of two genotypes of Bordetella pertussis during a period of increased

3 pertussis activity in Alberta, Canada: January to August 2012. Int. J. Infect. Dis. 29:223-5.

4

5 Smith, T., Rotondo, J.,Desai, S., and Deehan, S. 2014. Pertussis surveillance in Canada: trends to 6 2012. Canada Communicable Disease Report. 40:21-30.

8 Tsang, R.S., Sill, M.L., Advani, A., Xing, D., Newland, P., and Hallander, H. 2005. Use of 9 monoclonal antibodies to serotype Bordetella pertussis isolates: comparison of results obtained 10 by indirect whole cell enzyme-linked immunosorbent assay and bacterial microagglutination 11 methods. J. Clin. Microbiol. 43(5):2449-51.

13 Tsang, R.S., Shuel, M., Jamieson, F.B., Drews, S., Hoang, L., Horsman, G., Lefebvre, B., Desai, 14 S. and St-Laurent, M. 2014. Pertactin-negative Bordetella pertussis strains in Canada:

15 characterization of a dozen isolates based on a survey of 224 samples collected in different parts 16 of the country over the last 20 years. Int. J. Infect. Dis.28:e65-9.

17

18 van Gent, M., Heuvelman, C.J., van der Heide, H.G., Hallander, H.O., Advani, A., Guiso, N., 19 Wirsing von Konig, C.H., Vestrheim, D.F., Dalby, T., Fry, N.K., Pierard, D., Detemmerman, L., 20 Zavadilova, J., Fabianova, F., Logan, C., Habington, A., Byrne, M., Lutynska, A., Mosiej, E., 21 Pelaz, C., Grondahl-Yli-Hannuksela, K., Barkoff, A.M., Mertsola, J., Economopoulou, A., He, 22 Q., and Mooi, F.R. 2015. Analysis of Bordetella pertussis clinical isolates circulating in 
1 European countries during the period 1998-2012. Eur. J. Clin. Microbiol. Infect. Dis. 34(4):821-

230.

3

4 Varughese, P. 1985. Incidence of pertussis in Canada. Can. Med. Assoc. J. 132(9):1041-2.

5

6 Winter K, Harriman K, Zipprich J, Schechter R, Talarico J, Watt J, et al. 2012. California

7 pertussis epidemic, 2010. J Pediatr. 161(6):1091-6.

8

9 World Health Organization SAGE pertussis working group. 2014. Background paper. Geneva;

$10 \quad 2014,82 \mathrm{p}$.

11

12 World Health Organization. 2015. Pertussis Vaccines: WHO Position Paper - August 2015.

13 Wkly Epidemiol Rec. 90(35):433-60.

14

15 Zeddeman, A., van Gent, M., Heuvelman, C.J., van der Heide, H.G., Bart, M.J., Advani, A.,

16 Hallander, H.O., Wirsing von Konig, C.H., Riffelman, M., Storsaeter, J., Vestrheim, D.F., Dalby,

17 T., Krogfelt, K.A., Fry, N.K., Barkoff A.M., Mertsola, J., He, Q., and Mooi, F. 2014.

18 Investigations into the emergence of pertactin-deficient Bordetlla pertussis isolates in six

19 European countries, 1996 to 2012. Euro. Surveill. 19(33):pii20881.

20

21 
Table 1. Sequence type distribution of 53 Bordetella pertussis isolates from Quebec, Canada.

\begin{tabular}{lccc}
\hline Sequence & \multicolumn{2}{c}{ Number of Isolates (\%) } & \\
Type (ST)* & $2002-2006$ & $2007-2014$ & Total \\
\hline ST-1 & $23(92.0)$ & $11(40.7)$ & 34 \\
ST-2 & $1(4.0)$ & $11(40.7)$ & 12 \\
ST-4 & $0(0)$ & $1(3.7)$ & 1 \\
ST-7 & $1(4.0)$ & $0(0)$ & 1 \\
ST-8 & $0(0)$ & $4(14.8)$ & 4 \\
\hline Total & 25 & 27 & 52 \\
\hline
\end{tabular}

*ST-1: ptxS1A, prn2, fim3B, fhaB1, ptxP3; ST-2: ptxS1A, prn2, fim3A, fhaB1, ptxP3; ST-4: ptxS1A, prn1, fim3A, fhaB1, ptxP1; ST-7: ptxS1A, prn3, fim3B, fhaB1, ptxP3; ST-8: ptxS1A, prn9, fim3B, fhaB1, ptxP3. 